\title{
Sobre las élites y el poder de clase en El balneario (1986) y El premio (1996), de Manuel Vázquez Montalbán
}

\author{
CAROLINA FERNÁNDEZ CORDERO \\ Universidad Autónoma de Madrid
}

En 1991, tras el éxito de Galíndez, la revista Quimera publica una entrevista a Manuel Vázquez Montalbán en la que se encuentran algunas claves importantes para entender el marco teórico sobre el que se desarrollan sus novelas:

\begin{abstract}
La literatura es un rito... incluso quien no ha querido intervenir directamente, también ha transmitido ideología, porque escribir siempre precisa una concepción del mundo y llevar a los libros cómo son los demás y cómo eres tú mismo, cuál es tu proyecto personal, aun cuando lo hagas en los códigos más herméticos (Padura 53).
\end{abstract}

Esta idea de la literatura como portadora de ideología resulta relevante en cuanto que el autor muestra conciencia de ella a la hora de construir y concebir los textos, lo que evidencia su compromiso no solo a través de su manera de interpretar el mundo sino también de su modo de transmitirlo. Los textos literarios de Vázquez Montalbán dotan a la literatura de un alcance que sobrepasa el pacto pasivo de ficción entre autor y lector para convertirse en un ejercicio de praxis ideológica.

Ya en los años 70, con sus escritos subnormales, encuentra en ella un espacio en el que experimentar estéticamente la denuncia de la realidad, elevando escritura y creación a la categoría de acto político, constante que le acompañaría con mayor o menor radicalidad a lo largo de toda su carrera. Poco después, superado este periodo, sus obras surgen como una enciclopedia diacrónica de la actualidad con punto de partida en la sociedad de la transición.

La crisis del petróleo de 1973, tras frenar los intentos aperturistas y la expansión económica de los años 60, devolvió a España a su lugar de origen, como consecuencia de su llegada — una vez más — tardía al desarrollo capitalista exterior que implicaba la modernidad (Balibrea 22).

Sin embargo, las clases sociales que habían posibilitado dicho crecimiento no accederían a alejarse de un poder al que tanto les había costado acercarse tras más de veinte años de dictadura. Es decir, llegado el 
momento de "modernizar" la economía española, las viejas oligarquías que habían monopolizado el poder hasta entonces se ven obligadas a aceptar a una nueva burguesía financiera emergente, portadora del capital que posibilitará la apertura al exterior. Esta será la que protagonice con el beneplácito del dictador hasta su muerte- junto con las anteriores la transición y la que encabece la sociedad hasta su desarrollo capitalista financiero en los años 90 . Se trataba de unas nuevas clases, como afirma Alfonso Ortí, que

debían ser capaces de articular un proyecto político que volviese a conciliar — tras la desaparición del Dictador - todas las fuerzas burguesas en torno a sus permanentes intereses objetivos últimos; es decir: en torno a las exigencias y límites determinados —en última instancia - por la reproducción de las relaciones de producción básicas del sistema capitalista — el despotismo de fábrica y la división en clases- y de las relaciones de poder — autoritarias y elitistas - que regulan y profundizan esta misma reproducción en el seno del Estado y de todo tipo de organizaciones jerárquicas (11).

La materialización de este proyecto político, la "ruptura pactada", dilató en el tiempo el poder de los diferentes órganos franquistas y cercenó la oportunidad de establecer una verdadera brecha entre los dos sistemas de gobierno. Las actuaciones - y declaraciones - de los protagonistas de la transición, desde Juan Carlos I hasta Carrillo, constatan que dicha "ruptura pactada" no fue sino una transacción de poderes políticos que dotaban de legitimidad a las clases hegemónicas anteriores —a pesar de las concesiones en otra dirección, como la legalización de los partidos obreros, pagadas, por otro lado, con la renuncia a sus teorías de base ${ }^{1}$.

La evidencia de que la democracia iba a ser comandada por fuerzas procedentes de la dictadura, sumada a los nuevos problemas consecuencia de la crisis social del momento, generó ese sentimiento de desilusión entre las izquierdas que se ha dado a conocer como "desencanto". De él surge la necesidad de darle dimensión política a la problemática social de la época, necesidad de la que a su vez nace la novela policiaca de Carvalho (Colmeiro, 1992: 32), donde Vázquez Montalbán encuentra, entre

\footnotetext{
-

${ }^{1}$ Para un análisis exhaustivo del momento histórico, cf. las obras de Morán, Gregorio. El precio de la transición. Barcelona: Planeta, 1991; Tusell, Javier y Soto. Álvaro (eds.). Historia de la transición (1975-1986). Madrid: Alianza Editorial, 1996; Grimaldos, Alfredo. La sombra de Franco en la Transición. Madrid: Oberon, 2004; y Gallego, Ferrán. El mito de la transición. Barcelona: Crítica, 2008, entre otros.
} 
otras muchas posibilidades, la de analizar la sociedad a partir de la división en clases, aunque siempre insertado en un marco más amplio que le impide profundizar como otros autores en el problema principal que surge de las tensiones creadas entre ellas. Lo que sí consigue sin embargo es seccionar, analizar y reflejar las contradicciones de una de ellas, la dominante, cuya evolución diacrónica se puede leer en dos de sus novelas, El Balneario (1986) y El premio (1996).

Aunque diferentes en sus tramas, tiempos internos y resoluciones del conflicto, comparten maneras básicas de estructuración y desarrollo que propician dos situaciones semejantes comparables a partir del análisis de clase.

El primer elemento común que permite dicha comparación viene dado a través la configuración de un espacio narrativo en el que se puede inmovilizar a los personajes paradigma de esa clase hegemónica que se va a analizar. Se trata entonces de llevar la escena y la trama al aislamiento - lo que le da impunidad frente a cualquier ataque externo-, de reducir la posibilidad de movimiento para impedir interferencias, añadidos o ficciones casuales.

Así, el espacio en que se ubica la trama de la novela de 1986, el que da nombre a la obra, se presenta como un lugar aislado del mundo, un espacio de reposo, de descanso, un rincón donde esconderse del vértigo de la realidad, un balneario que posibilita la huida de la cotidianeidad lejos de la modernidad de las ciudades. Por el contrario, la novela de 1996 exige un espacio de aislamiento más (pos)moderno: un "Madrid Manhattan” y un hotel de lujo de estilo "posmariscal heavy"”2.

También la posición en la que el autor ubica a Carvalho es compartida por las dos novelas, ya que en ambos casos funciona casi como un espectador más, alguien que observa entre bastidores, a la sombra de los órganos oficiales, con menor implicación activa que en otros relatos. En

\footnotetext{
${ }^{2}$ Resulta fundamental para la comprensión de la evolución de un espacio a otro la descripción de la ciudad de Madrid en El premio: "una ciudad de un millón de chalecos en aquella Transición dirigida por jóvenes ejecutivos que se ponían chalecos para sentirse más vertebrados. Luego los socialistas se quitaron los chalecos y los que llegaron al poder descubrieron las camisas de marca. Ahora observo el regreso de algunos chalecos. Volverían pronto las derechas al poder. Madrid se había convertido en la ciudad del millón de dossiers, donde todo el mundo trafica con lo que sabe sobre las cloacas ajenas” (74-75).
} 
El Balneario aparece como un cliente cualquiera, de repente envuelto profesionalmente en la trama principal —una justificación narrativa para dar verosimilitud al curso de los hechos. Y en El premio, aunque contratado desde el comienzo como detective, tampoco era para resolver el caso del asesinato con el que se encuentra, de modo que el acostumbrado papel de investigador protagonista pasa a un plano secundario. Sin embargo, a cambio del desplazamiento de Carvalho, Vázquez Montalbán consigue llevar el foco a la formación de colectividades y configurar un exhaustivo análisis de la clase hegemónica de los años en que se ubica la narración.

Un tercer elemento de cohesión desde el que el autor configura la clase social hegemónica viene dado por el tratamiento de la gastronomía. Tanto en una como en otra las élites se ven definidas a partir del ayuno o de la prisa que impide la dedicación al tiempo de comida. La gastronomía de los ricos, definida por el "no aumentar de peso y no consumir grasas” que ya se podía leer en Los mares del Sur (Saval 389-400), se lleva al extremo en El Balneario, donde la comida se limita a "una taza de caldo vegetal con perejil al mediodía y un vaso de zumo de frutas al anochecer", así como a "la obligación, desde luego moral, de beber al menos dos o tres litros de agua al día” (13), y vuelve a definirse como característica de clase en El Premio —recuérdese, por ejemplo, el menú propuesto por Lázaro Conesal para su comida con el Ministro: "Un zumo de pomelo y un filete, vuelta y vuelta” (169).

Los dos últimos aspectos descritos muestran el interés del autor por anteponer la realidad social del momento a la investigación del caso, núcleo de la ficción del texto. De este modo, subvierte la finalidad escapista propia del género, para convertirlo en "un escapismo que postula la realidad como extremo fantástico, para el que el enigma — superficial, indiferente, incluso banal - es un mero pretexto y un punto de partida", en un "escapismo invertido", según palabras de Joan Ramón Resina (262-263).

En los casos de las novelas analizadas, será la clase hegemónica la realidad social elegida por el autor, hasta cristalizarla para someterla a un proceso histórico social que demuestra su evolución y desarrollo.

Así, en primer lugar, la sociedad que crea Vázquez Montalbán en ese espacio cerrado de El Balneario responde a la más básica organización 
empresarial, elemento de la modernidad capitalista: mandos de una empresa (médicos, jefes de la administración, etc.), subalternos y clientes.

La cúpula directiva del balneario se presenta a priori como modelo de una sociedad europea más desarrollada, pero al desvelarse la trama, se descubren como antiguos fascistas que en los años de franquismo (y también posteriormente) encontraron en España el paraíso del silencio que los ampararía.

El más relevante de ellos, el que abre la novela, el último asesinado, el relator de toda la historia de los directivos, responde al nombre de Gastein, el médico principal, un suizo, mano derecha de los propietarios de la instalación, los Hermanos Faber, dos ávidos empresarios, que con tal de hacer riqueza accedieron a vender su dignidad a dos hermanas bielorrusas anticomunistas tras la II Guerra Mundial. Una de ellas, cuya función en el balneario es la de relaciones públicas, aparece siempre descrita — no con poca ironía — como una monja (Madame Fedorovna o Catalina Ostrovsky) y la otra, que figura en calidad de cliente, como una anciana bigoréxica (Mistress Simpson o Tatiana Ostrovsky). Sin embargo, las dos esconden bajo su aparente "buena salud" mental y física no solo negocios de la Guerra Fría sino también el archivo de las SS bielorrusas y el Gobierno, además de riquezas confiscadas en la URSS y en Polonia. La historia de sus relaciones con los tres anteriores y su consecuente exterminio endogámico configura la trama de la novela, trama que nada casualmente ha sido calificada por Gustavo Rodríguez-Morán como "una repetición del ángel exterminador que acaba devorando a su propia sociedad” (95, n. 3).

Frente a ellos se sitúan los clientes — sin incluir a la falsa Mistress Simpson-, el elemento social que define al balneario como "la reserva espiritual de lo más selecto de la vieja derecha española” (239); ellos son los representantes de esa clase social dominante mixta y moderna:

Ciudadanos económica u socialmente de primera, pero pobre y débil gente incapaz de luchar cotidianamente contra las tentaciones o dotados de un código genético desconectado de la moderna cultura del aspecto, posterior a los años de la reconstrucción mundial en la que también jugó su papel la recuperación desmedida de grasas, proteínas y vitaminas (11-12).

O lo que es lo mismo: 
La nueva clientela de ricos gordos o intoxicados por los malos hábitos de vida de la modernidad. Alemanes, suizos, franceses, belgas y también obesos españoles de la zona del dólar madrileño o del marco catalán (13).

Sus posiciones, alianzas, enfrentamientos y subdivisiones ante el conflicto de saber quién es el asesino y cuándo van a poder salir del aislamiento impuesto desde el primer asesinato, hacen del balneario un mapa de Europa, como indica la cita que encabeza el libro. En esas posiciones hay una voluntad de Manuel Vázquez Montalbán de separar a los españoles del resto de los europeos, muestra de una sociedad inmadura, demasiado arraigada todavía a su historia precedente, un detalle nada casual teniendo en cuenta, por un lado, que se trata de las élites del país, y por otro, que lo hace en un momento en que España acaba de ratificar su entrada en la OTAN y su adhesión a la CEE.

Alianzas y divisiones aparte, el episodio más representativo en la simbolización de ese trasvase de poderes que fue la transición es la llamada "Operación Hipercalórica" precedente al primer asesinato. Una misión absurda ya desde el nombre mismo, que consiste en asaltar la cocina y conseguir el elemento prohibido, la comida. Esa es la subversión y contradicción ridícula de la clase alta, que genera motu proprio su ayuno contra el que después se rebela como si de un acto heroico se tratara.

En ella interviene lo más representativo de los poderes fácticos en la realidad del momento, en cuyo frente se alza un militar que se jacta de su condición de militar e incluso habla y actúa como tal, a pesar de que dejó de serlo a efectos económicos cuando el Gobierno le quiso quitar la fábrica de piensos que tenía. El coronel don Ernesto Villavicencio, "sesentón, brazicorto, tobillos de elefante y un corpachón de mozo de cuerda al servicio de un corazón de coronel jubilado del arma de Infantería” (21), se presenta como el cerebro de la operación. Su momento de gloria en el balneario lo encuentra, por un lado, en llevar el mando de la hazaña y, por otro, en atreverse a revelarle su condición de militar "porque la conciencia del ejército es universal, que un militar es militar mientras vive" (49), afirma él mismo - a un general belga del que no se separa. Sin embargo, según otro de los personajes, tiene, sin más, pinta de "cabo chusquero" (56).

Villavicencio convoca para esta misión a "hombres bregados que no se arruguen ante las dificultades y que estén dispuestos a todo sacrificio 
premiado con un suficiente botín” (55): Telmo Duñabeitia, “industrial vasco con $15 \mathrm{~kg}$ de más" repartidos "por un cuerpo macizo de descendiente de aizkolari que puso una serrería a tiempo e inició una dinastía que le pesaba a Duñabeitia como una montaña sagrada”. Tosco en exceso, disfrutaba disputándose la responsabilidad de la prosperidad del país con Juanito Sullivan Álvarez de Tolosa, "señorito de Jerez que proponía ser llamado Sullivan a secas”. Sin embargo —un dato nada desdeñable—, no era Duñabeitia el preferido de esta representación de la aristocracia. Ese puesto lo ocupaba "un elevado otoñal con bigotillo de ex funcionario franquista, siempre por delante o por detrás de su mujer, enjuta como un personaje femenino y castellano de novela nacionalista de los años cuarenta”, el hombre del chándal, que a su vez estaba obsesionado con un "escritor gordo y taciturno al que sabía vinculado al PC”, el escritor Sánchez Bolín, un personaje recurrente de las novelas de Carvalho, siempre calificado como alter ego de Vázquez Montalbán ${ }^{3}$.

Al general retirado, al empresario vasco y al señorito andaluz hay que sumarles un tercer hombre, "un joven de muchas carnes, fugitivo de algún relato manchego convencional, cejijunto aunque ojiabierto, con mucho pelo negro a manera de boina y boca sensual de descendiente de arrieros mamones de todo lo bueno, paticorto, pero patirrecio y con un tórax de Sansón celtíbero", Tomás Sánchez Pérez, propietario único de una red de queserías, con la principal en Madrid y que da nombre a su linaje, Quesos Sánchez Pérez Hnos. e Hijos. Su estatus, no obstante, queda invalidado por su edad en este balneario, donde las antiguas jerarquías y sus valores se respetan por encima de toda novedad social:

Excesivamente joven para tanta responsabilidad y riqueza, ninguno de los jefes naturales de la camada adelgazante le reconoce la jerarquía y el coronel le trata poco menos que como a un ordenanza todo terreno (26).

A estos cuatro se les suma Carvalho, que de nuevo funciona simplemente como justificación narrativa para dar un sentido a la escena en el desarrollo de la trama.

Las relaciones entre este grupo serán estrictamente militares y comandadas por el coronel Villavicencio. Con un plan perfectamente delimitado, en cuya confección nadie participa con seriedad excepto el coro-

\footnotetext{
${ }^{3}$ Para las descripciones completas de estos personajes, cf. 22-23.
} 
nel, ejecutan su operación, que se salda con el inmenso saqueo de una manzana y un "prisionero de guerra". Suficiente para el coronel Villavicencio, orgulloso de su hazaña, como muestra en una arenga aprendida sobre la tipología del valor, que finaliza con una nostálgica evocación a Franco. Un momento de gloria interrumpido no poco sintomáticamente por el actual aparato represor de la democracia, el guardia de seguridad, que con un "acabemos ya el cachondeo", deporta al comando de nuevo al tiempo presente mientras Villavicencio apela al honor y se acoge a las leyes militares (71-72).

Este comando grotesco y caricaturizado es solo un ejemplo de comportamiento por parte de un grupo formado por hasta veinticinco clientes con nombre y apellidos. Su autonomía narrativa, que lo aleja en parte de la trama principal, dota al episodio de una interés particular, y no solo porque sea protagonizado por los máximos representantes de la élite de la época — dos empresarios (viejo y joven), un militar y un aristócrata-, sino también por las relaciones de poder que entre ellos se establecen, pues aunque sea narrado en clave de humor, finalmente todos han asumido el mando del militar sin ninguna dilación.

La evolución de este paradigma de la élite y de sus relaciones de poder en los años venideros es la que se puede leer en otra novela de contexto similar, El premio, concebida, escrita y publicada en plena decadencia socialista, protagonizada por el GAL y la corrupción política. El inminente regreso de la derecha al poder obliga a Manuel Vázquez Montalbán a una recolocación en la praxis literaria de su ideología, que opta por la radicalización mediante la denuncia del capitalismo salvaje, máximo emblema del neoliberalismo recién inaugurado ${ }^{4}$. Así, en una entrevista de José F. Colmeiro en 1996, antes de las elecciones, vuelve a plantear el problema del poder de las clases hegemónicas como objeto de denuncia:

Si en el siglo XIX el avance democrático se hizo para luchar contra un determinado tipo de élites condicionadas por la herencia y el dinero, básicamente, en estos momentos el ideal de alcanzar una democracia representativa y ricamente participativa también se tendría que hacer contra unas élites, pero que ya no son las mismas; suelen ser las élites de los instalados dentro del grupo social emergente, que no tienen

${ }^{4}$ No es casual, por tanto, que comparta años de publicación con la biografía de Dolores Ibárrruri (Pasionaria y los siete enanitos) y el análisis político de la Europa de los 90 en Panfleto desde el planeta de los Simios. 
que ser solamente los que tienen el dinero, sino también los que tienen poder informativo, etc., y algunos se corresponden a la aristocracia que delega una oligarquía del siglo pasado y la adapta a la nueva época. En estos momentos lo que llamábamos antes oligarquía, que es el gran enemigo de la democracia participativa, es otra cosa más compleja, completamente internacionalizada (280).

Por tanto, ya no interesan las viejas estructuras de la transición, ahora el foco recae en estas nuevas élites capitalistas, neoliberales, relevo de esas oligarquías dominantes retratadas diez años atrás.

Si en El Balneario las relaciones se definían en términos de poder bajo el colectivo clientes, en El premio ese poder se va a ver monopolizado en una única persona en torno a la que van a girar todos los personajes que conforman la trama: Lázaro Conesal, el Gran Gatsby, el plutócrata preferido de la élite española, que tras conseguir el control de todas las operaciones financieras del país, ve peligrar sus privilegios ante la celebración de las elecciones del momento. Toda la obra se resuelve como un esfuerzo por configurar este patrón de poder, tanto desde sus propias acciones como desde los elementos que se ubican a su alrededor. De todas las definiciones que Vázquez Montalbán incluye, la más ilustrativa viene de un figurante Juan José Millás que le explica a Pepe Carvalho:

Conesal es el emblema de los nuevos ricos del nuevo régimen democrático. El self made man que trafica con las mejores influencias y sorprende a los tiburones fingiendo el lenguaje del delfín y a los delfines mordiéndoles como un tiburón (89).

Su personalidad y su retrato de feroz financiero quedan registrados en la conversación que le concede a dos chicas para una "monografía sobre las actitudes del poder financiero en España", en la que, mediante una descripción de lo que es el capitalismo, teoriza toda su acción y su imperio de bienes:

\footnotetext{
¿Por qué ha de ser humano el capitalismo? ¿Qué es lo humano, señoritas? Navidad y los villancicos. Pero el capitalismo no tiene por qué ser humano, ni tener otra ética que la eficacia de la razón orientada hacia la acumulación de un máximo beneficio en las manos más responsables. Los escándalos y las crisis se corresponden a la naturaleza del capitalismo, son la regla, no la excepción. La especulación y la cultura del pelotazo, tan cínicamente condenadas por todos los que la promueven y se benefician de ella, corresponden al corazón del sistema (166).
} 
El capitalista, desposeído de una ideología que no sea la del propio capital, muere y resucita como el ave fénix, se sabe uno de los pilares del sistema y se defiende ante cualquier amenaza que desde otras posiciones consciente o inconscientemente se le presenten delante:

Voy a poner un ventilador ante toda la mierda que conozco y aquí no se salva ni Dios, ni siquiera ese estúpido gobernador del Banco de España que obra al diktat de todas las mafias del poder y los señores del dinero. Estos socialistas de pacotilla se cagan ante los señores del dinero. He conseguido ser lo que quería ser para que ahora venga esa colección completa de derrotados a llevarme con ellos a su tumba política. Cuando pierdan el poder no serán nada y en cambio yo me reharé de esta puñalada por la espalda y bailaré sobre sus esqueletos de cabrones. Dentro de unos meses, cuando ganen las derechas, toda esa gentecilla aupada sobre los tacones postizos del poder político serán cesantes, miserables cesantes que deberían volver a su mediocre existencia anterior y muchos de ellos ni eso. Entonces los iré recogiendo con una pala mecánica y los tiraré al vertedero más asqueroso de Madrid o les iré metiendo billetes de cinco mil pesetas en la boca hasta que revienten y los saquen por el culo (273).

Su mujer, Milagros Jiménez Fresnedo, lo compara con Atila — por donde pasa no crece la hierba - y su control sobre la clase política es tal que, como él mismo afirma, sabe "incluso si follan con preservativo o si se las menea un chimpancé” (274).

Sin embargo, el poder de Conesal no se mantiene gracias y exclusivamente a su posición como sujeto individual, sino que está amparado por una red social tejida en todo este tiempo de gobierno socialista, cuyo puesto de honor lo ocupa un hijo resignado, cansado de las maneras de su padre, pero al fin y al cabo heredero con sumisión del emporio que le aguarda. De su hijo para abajo se establece la manada de buitres que lo sobrevuelan, encabezada por sus dos socios, Iñaki Hormazábal y Celso Regueiro Souza.

Regueiro Souza, “chatarrero y propietario de avionetas de alquiler, íntimo del jefe del Gobierno, fuera el que fuese, al que se dirigía incluso dándole la espalda” (33), autodefinido como parte de la beautiful people y emparentado sexualmente con Álvaro Conesal, el cual asegura que "aportó al grupo sus buenas relaciones con el Gobierno que nos permitieron pujar por empresas reprivatizadas a precios de ganga, pero ha salido implicado en demasiados líos de corrupción y se hunde con el Gobierno" (191). 
Mucho más agresivo y seguro se presenta Iñaki Hormazábal, “el calvo de oro, para las damas de todo Madrid o el asesino de la telefónica, denominación merecida por su manía de comprar, matar, desguazar, vender empresas por teléfono" (33). Su facilidad para asociarse sin escrúpulos con quien más le interese le ubica en una posición mucho más optimista y adaptada a las circunstancias financieras producidas en ese tiempo de declive socialista.

No se equivocaba la mujer de Conesal cuando le decía a Álvaro que su padre siempre estaba "rodeado de fascistas, explotadores y putas", como se puede comprobar en la selección de la élite descrita entre los 500 invitados que asisten esa noche al premio. Se trata de "gentes de letras, ricos de diferentes lugares y políticos (estos los menos)", como explica el propio Conesal (185). El conjunto de todos ellos forma parte de la novela desde el comienzo pero cobran mayor sentido a partir de la muerte del empresario, es decir, cuando se convierten en sospechosos.

La división tripartita —o trinitaria, tal vez — de gentes de letras, ricos y políticos se corrobora en la distribución por mesas, establecidas de mayor a menor antigüedad en el poder.

En la mesa de mayor rango se encuentran sus dos socios y la tradicional aristocracia. Entre los últimos destaca el duque de Alba, antes y para los que entonces lo conocieron, Jesús Aguirre y Ortiz de Zárate, que se toma la pedantería de dividir a sus compañeros, en un homenaje a la obra de Umberto Eco, en apocalípticos e integrados, a la luz de un discurso en forma de "collage mental del ex jesuita, capaz de mezclar a las genealogías más necias de la aristocracia española superviviente, con las genealogías de la escuela de Frankfurt o del mismísimo György Lukács” (33).

Entre los apocalípticos, los pesimistas frente a la situación actual, se encuentran - no con poca ironía- los casi arruinados Hormazábal y Regueiro, y entre los integrados, los que se toman la actualidad con optimismo, los que siempre conservan su estatus en la élite, los aristócratas Beba Leclercq, "de los Leclercq de Tejados y Demoliciones, una rubia elástica y dorada”, y su marido Sito Pomares, de Pomares \& Ferguson, "bodegueros de Jerez, un rubicundo cuadrado y pecoso" (33-34), un "pedazo de carne bautizada y confirmada en las iglesias del Opus Dei”, “del sector más rico pero también más tonto”, según Álvaro Conesal. Pomares nunca estableció una relación demasiado buena con el empresario, pero tampoco mala, porque según este, "tras veinte años de descanso histórico tras la muerte de Franco, su gran celestina, vuelven a la carga” 
(97) y no resultaría estratégicamente inteligente rechazarlo si quiere mantener controladas todas las ramas del poder. Ella, sumisa e ignorante, degradada al extremo por Vázquez Montalbán en detalles como cuando apela "a ese ruso, Lucas" para referirse al filósofo húngaro, se descubre como la amante preferida de Conesal en un romance consentido por el marido hasta que trascienden las apariencias - momento de la novela-, en que él se ve obligado a defender su honra, un gesto muy acorde con su protocolo social (250-254).

En la segunda mesa se encuentra Jorge Justo Sagazarraz, heredero de una empresa naviera de capital mixto, especializada en la fabricación de pesqueros dedicados a la pesca del calamar —el colmo de la división del trabajo-, antes fructífera, ahora en pleno hundimiento. Sagazarraz se autodefine como una "vieja joven promesa de empresario" y su tragedia es la de "un naviero casi arruinado, bueno, arruinado, en declive", despechado por el desinterés de Conesal. Junto a él aparece Mona d’Ormesson de los Fresnos de Ruiseñada, hija de Pocholo d'Ormesson, prima de la condesa de los Cantos, amante de Paco Umbral y de Unión de Explosivos de Riotinto, si se atiende a la descripción del duque de Alba, porque el autor la presenta simplemente como "traficante de influencias intelectuales y traductora en sus horas libres” (28). Invitada por Conesal para ejercer "como puente entre el poder institucional cultural y la beautiful people lectora" (186), hace lo propio con otro comensal, el "académico especializado en el diminutivo del siglo XVII" —otro colmo de la especialización-, Mudarra Daoiz, obsesionado por negarse a todo lo catalán "mientras persista en Cataluña el genocidio contra la lengua española" (131), así como con un ya consagrado y posmarxista Sánchez Bolín, cuya dialéctica reside en ser comunista alimentado de las élites hegemónicas.

Otra mesa, aunque ya de menor rango social, es en la que se sientan el Sr. y Sra. Puig, de Puig S.A., fabricantes de sanitarios. Llave de paso de Conesal para los círculos catalanes, el Sr. Puig se define como muy lector en su juventud — se jacta de haber leído la trilogía de Gironella sobre la guerra civil...-, pero no interesado en la literatura. En esta ocasión custodia su estatus ante los cambios políticos posibles, puesto que los socialistas necesitan a CiU y los votos de estos dependen en última instancia de los empresarios catalanes (223). A estos dos se les suma un cuarteto representante del sector de los escritores, caricaturizados en extremo, pujantes por el premio: Andrés Manzaneque, "el mejor poe- 
ta y novelista gay [de Cuenca] de su generación en las dos Castillas, apreciación no aceptada por los mejores poetas y novelistas gays de León, que rechazaban mayoritariamente la unidad político-administrativa autonómica formada por Castilla la Vieja y León” (26), presentado al premio manipulado por la asesora editorial Marga Segurola; Alma Pondal - Mercedes en su vida privada—, "la mejor novelista ama de casa", presentada al premio con una novela inexistente por orden del crítico literario Lorenzo Altamirano; Oriol Sagalés, "una de las eternas promesas de la literatura, capaz de haber llegado a los 50 años con un número limitadísimo de lectores selectos de los que conocía sus números telefónicos e incluso de las segundas residencias" (16). Su desorden mental y su histeria llegan hasta el punto de declararse absurdamente culpable de la muerte de Conesal; y Ariel Remesal, "representante de la mesocracia letraherida, los escritores corporativizados...", amigo de Regueiro Souza, escritor de Ouroboros, el texto que merodea constantemente en la atmósfera de la obra para reproducir muy acertadamente el mito del eterno retorno.

El elenco de estos "letraheridos” lo cierran los máximos poderes fácticos del mercado literario: Marga Segurola, "experta literata que ejerce de consultora de editoriales, españolas y extranjeras, revistas literarias, programas culturales de radio y televisión”, perfecta conocedora de todos los mecanismos del mercado literario, encargada de componer toda la trama comercial y mercantil del premio junto con Conesal y Lorenzo Altamirano, "el crítico más reputado de toda España”, consejero literario personal del financiero, responsable de la pantomima literaria -incluida la composición del falso jurado- , a pesar de su profunda fe en el valor emblemático de la literatura (210-211).

En un lugar aparte o en ningún lugar concreto, realmente, sitúa Vázquez Montalbán a los políticos Joaquín Leguina, presidente de la Comunidad de Madrid, y Carmen Alborch, Ministra de Cultura, relegados al puesto de informadores intermediarios entre la policía y los invitados, en una clara ejemplificación del lugar que ocupa realmente la clase política en la democracia del momento.

A medida que va avanzando la novela en esa estructura de serpiente -parte del mito del ouroboros-, oscilante del pasado al presente en el tiempo, todos estos personajes van cobrando protagonismo compartido con el asesinado- como colectivo en la trama, hasta configurarla en torno a las relaciones entre todos ellos primero y después con 
Lázaro Conesal. Su descripción en la narración los categoriza como elementos orgánicos del poder del financiero, cuyo desarrollo social se compara de nuevo y esta vez incluso en la propia novela con El ángel exterminador de Buñuel (142).

Por tanto y a partir de las descripciones expuestas en estas dos novelas, parece claro que existe un interés ideológico por parte de Manuel Vázquez Montalbán de reflejar mediante la experiencia estética la evolución de la categoría hegemónica y el trasvase de poderes de un tipo de sociedad recién salida de una transición heredera de las estructuras franquistas a una sociedad absorbida por el llamado "capitalismo salvaje”; es decir, de una élite que lucha una vez más por entrar en la modernidad y otra que, sin completar el ciclo anterior, se intenta adaptar a las exigencias de la posmodernidad.

\section{Bibliografía}

Balibrea, Mari Paz. En la tierra baldía: Manuel Vázquez Montalbán y la izquierda española en la posmodernidad. Barcelona: El Viejo Topo, 1999.

Colmeiro, José F. "En busca de los imaginarios perdidos: Entrevista con Manuel Vázquez Montalbán.” Manuel Vázquez Montalbán: el compromiso con la memoria. Coord. José F. Colmeiro. Woodbridge: Tamesis, 2007: 279-296.

_. "Posmodernidad, posfranquismo y novela policiaca.” España Contemporánea V, 2 (otoño 1992): 27-40.

Gallego, Ferrán. El mito de la transición. Barcelona: Crítica, 2008.

Grimaldos, Alfredo. La sombra de Franco en la Transición. Madrid: Oberon, 2004.

Morán, Gregorio. El precio de la transición. Barcelona: Planeta, 1991.

Ortí, Alfonso. "Transición postfranquista a la Monarquía parlamentaria y relaciones de clase: del desencanto programado a la socialtecnocracia transnacional." Política y Sociedad 2 (1989): 7-20.

Padura Fuentes, Leonardo. "Reivindicación de la memora. Entrevista con MVM.” Quimera 106-107 (1991): 47-53.

Resina, Joan Ramón. "Desencanto y fórmula literaria en las novelas policíacas de MVM." MLN 108.2 (marzo 1993): 254-282.

Rodríguez-Morán, Gustavo. "Del bien comer y del bien escribir. Desestabilizando cánones culturales en la serie Carvalho.” Manuel Vázquez 
Montalbán: el compromiso con la memoria. Coord. José F. Colmeiro. Woodbridge: Tamesis, 2007: 91-104.

Saval, José V. "La lucha de clases se sienta a la mesa en Los mares del sur.” Revista Hispánica Moderna 48.2 (dic. 1995): 389-400.

Tusell, Javier y Soto. Álvaro (eds.). Historia de la transición (19751986). Madrid: Alianza Editorial, 1996.

Vázquez Montalbán, Manuel. El premio. Barcelona: Planeta, 2008.

_. El balneario. Barcelona: Planeta, 1986. 